Z Herz- Thorax- Gefäßchir 2016 · 30:73-74 DOI 10.1007/s00398-016-0067-4

Online publiziert: 23. März 2016

๑) Springer-Verlag Berlin Heidelberg 2016

CrossMark

K. Kallenbach

Haerzzenter - INCCl, Luxembourg, Luxembourg

\title{
Publikationspreis der Zeitschrift für Herz-, Thorax- und Gefäßchirurgie
}

den jeweiligen Rubriken publiziert werden. Eine Listung der Zeitschrift in Medline oder sogar die Bewertung mit einem Impact-Faktor wird mit diesem Konzept nicht erfolgen können. Damit fehlt allerdings auch ein wesentlicher Antrieb, einen Beitrag zur Veröffentlichung an die Zeitschrift zu senden, da der Name weder in Medline auftaucht noch ImpactPunkte für die Karrierebildung gesammelt werden können.

Daher hat sich das Herausgebergremium der Zeitschrift überlegt, einen Preis auszuloben, der jeweils den besten Beitrag eines Jahres, der in der Zeitschrift veröffentlicht wurde, prämiert. Dieser Preis ist verbunden mit einem nicht unerheblichen Preisgeld von 2500,- €, das gemeinsam von der DGTHG und dem Springer-Verlag aufgebracht wird. Somit ist es Ziel des Preises, die Autoren dieser bereits fertiggestellten Artikel für ihre Beiträge zu belohnen und gleichzeitig potenzielle Autoren zu motivieren, ein Manuskript an die Zeitschrift für Herz-, Thorax- und Gefäßchirurgie einzureichen. Und da nicht alle Autoren mit einem Preis belohnt werden können, wird fairerweise der beste Beitrag prämiert.

Die Auswahl des Preisträgers erfolgt nach einem transparenten Verfahren: Alle Rubrikherausgeber der Zeitschrift dürfen aus dem abgelaufenen Kalenderjahr einen Beitrag aus ihrer eigenen Rubrik oder aber, wenn sich hier kein preiswürdiger Beitrag findet, auch einen Beitrag aus einer anderen Rubrik nominieren. Diese Nominierungen werden an alle Herausgeber verschickt, die dann die Artikel, die vom Springer-Verlag zur Verfügung gestellt werden, durcharbeiten und den aus ihrer Sicht besten Artikel benennen. Am Ende wird einfach zusammengezählt: Der Artikel, der die meisten Nennungen erhält, wird mit dem Preis belohnt. Sollte es zu einer Pattsituation kommen, erhält der federführende Schriftleiter 2 Stimmen. Natürlich entspricht es einem anständigen Umgang mit der Wissenschaft, das keiner der Herausgeber sich selber nominieren würde, wenn er Autor oder Koautor eines Beitrages wäre. Ich kann Ihnen daher versichern, dass der Herausgeber, der dieses Jahr als Koautor des ausgezeichneten Artikels fungiert, nicht seinen eigenen Beitrag vorgeschlagen hat. In diesem Jahr fiel übrigens das Urteil ziemlich eindeutig aus, der Beitrag von Herrn Al-Essawi wurde von der deutlichen Mehrheit als bester Artikel ausgewählt.

Liebe Leserinnen und liebe Leser, ich kann Ihnen unbedingt den Artikel von Herrn Dr. Al-Essawi, der den Publikationspreis 2015 erhalten hat, zur Lektüre empfehlen. Gleichzeitig hoffe ich, dass der neue Publikationspreis, der ja nicht ganz unerheblich pekuniär prämiert wird, Sie vielleicht dazu motiviert, Manuskripte bei der Zeitschrift für Herz-, Thorax- und Gefäßchirurgie einzureichen - ein kleines Dankeschön für ihren persönlichen Beitrag, die Mitglieder unserer Fachgesellschaft weiterzubilden!

Mit herzlichen Grüßen

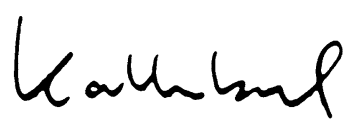

Prof. Dr. Klaus Kallenbach Federführender Schriftleiter 


\section{Korrespondenzadresse}

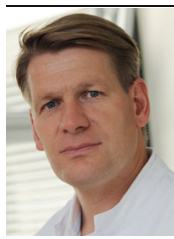

Prof. Dr. K. Kallenbach

Haerzzenter - INCCI

2 A rue N.E. Barblé,

1210 Luxembourg,

Luxembourg

kallenbach.klaus@incci.lu

Interessenkonflikt. K. Kallenbach gibt an, dass kein Interessenkonflikt besteht.

\section{Hirntod}

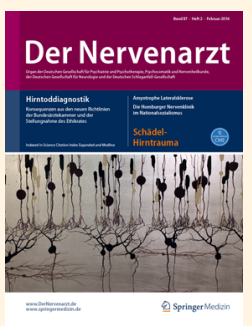

Seit Juli 2015

gelten für die Hirn-

toddiagnostik die

neuen Richtlinien

der Bundesärzte-

kammer in ihrer 4.

Fortschreibung.

Der Focus der

Zeitschrift Der Nervenarzt wurde daher in der Ausgabe 02/2016 auf den Schwerpunkt "Hirntod" gerichtet. Sie erhalten Informationen über die neuen Richtlinien und einen Überblick über den Stand der Diskussion zu diesem sensiblen Thema, das für alle Ärzte bei der Arbeit im Klinikalltag eine wichtige Rolle spielt.

- Kommentar zur Stellungnahme des Ethikrates

- Vierte Fortschreibung der Richtlinie zur Feststellung des irreversiblen Hirnfunktionsausfalls. Verfahrensabläufe und Neuerungen

- EEG und Evozierte Potentiale zum Irreversibilitätsnachweis der klinischen Ausfallssymptome des Gehirns

- Angiographische Verfahren zur Feststellung des zerebralen Zirkulationsstillstandes

- Organspende nach Herz- und Kreislauftod

Bestellen Sie diese Ausgabe zum Preis von 39,- EUR (zzgl. Versandkosten) bei Springer Customer Service Center Kundenservice Zeitschriften Haberstr. 7 69126 Heidelberg Tel.: +49 6221-345-4303 Fax: +49 6221-345-4229

E-Mail: leserservice@springer.com

Suchen Sie noch mehr zum Thema? Mit e.Med, dem Online-Paket von Springer Medizin, können Sie schnell und komfortabel in über 600 medizinischen Fachzeitschriften recherchieren.

Weitere Infos unter

springermedizin.de/eMed.

\section{Leitlinien zur Reanimation 2015 des European Resuscitation Council}

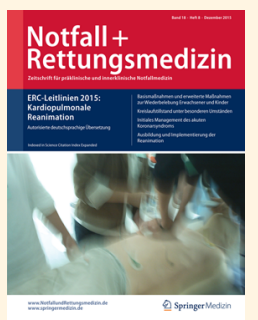

In der Ausgabe 8/2015 von Notfall+Rettungsmedizin wird zum vierten $\mathrm{Mal}$ die autorisierte deutschsprachige Übersetzung der

aktualisierten Leitlinien zur Reanimation des European Resuscitation Council (ERC) durch den Deutschen, den Österreichischen und den Schweitzer Rat für Wiederbelebung publiziert. Die Leitlinien geben die weltweit akzeptierte und auf höchstmöglichem Evidenzniveau basierende Sicht wieder, wie Reanimation wirkungsvoll und sicher durchgeführt werden kann.

Lesen Sie im Schwerpunktheft mehr zu folgenden Themen:

- Basismaßnahmen zur Wiederbelebung

- Erweiterte Reanimationsmaßnahmen für Erwachsene

- Kreislaufstillstand in besonderen Situationen

- Postreanimationsbehandlung

- Lebensrettende Maßnahmen bei Kindern

- Versorgung und Reanimation Neugeborener

- Initiale Management des akuten Koronarsyndroms

- Erste Hilfe

- Ausbildung und Implementierung der Reanimation

- Ethik der Reanimation und Entscheidungen am Lebensende

Bestellen Sie diese Ausgabe zum Preis von 39,- EUR zzgl. Versandkosten bei Springer Customer Service Center, Kundenservice Zeitschriften Tel.: +49 6221-345-4303

E-Mail: leserservice@springer.com 